\title{
EDITORIAL
}

\section{LATE COMPLICATIONS OF TRAUMATIC PARAPLEGIA}

THE effective early treatment of spinal cord injured persons, developed during the era of the Second World War, led to a dramatic reversal of mortality. As a consequence of survival, a large number of these seriously handicapped persons have returned to the community and entered the field of competitive living with energy and enthusiasm in pursuit of vocational or avocational goals. Thus, we have had the opportunity of observing a significant number of such persons for up to a generation. This exposure has brought to light certain late complications deriving from a variety of causes, not all of which have been identified. However, they do fall roughly into several categories.

The first group became manifest when patients complained of pain in the upper extremities and this disability was recognised as being related to the excess of stress and strain imposed on the arms by the changed life-style whereby double stress was imposed on these members whether the person was wheelchair bound or ambulatory with crutches or canes. While the specific cause of the pain in each case may not readily be identified, the pain and disablement resulting from rupture of the biceps or triceps tendons and the instability of neurotropic joints were readily diagnosed. No more are the trunk and lower extremities exempt particularly in the incomplete ambulatory person. Neurotropic joints have been recognised in the vertebral column, the hip and the knee. Pain in the back and lower limb joints is frequently reported.

Chronic swelling of the lower extremities is a recognised concomitant of lower limb dependency particularly in the person with a lower motor neuron lesion whether wheelchair bound or ambulatory. The state of nutrition of the tissues deteriorates and simple abrasions may lead to ulceration necessitating amputation to prevent the central spread of infection.

Of similar origin is the development of haemorrhoids and rectal prolapse due to the combination of trunk dependency and repeated straining for evacuation. This, in turn, tends to result in a degree of obstruction to the passage of stool; a vicious circle.

The second category is of particular concern for while the early lethal complications are capable of being prevented, prolonged exposure to the spinal cord injured state permits some of them to creep back in. Thus, a small contracted bladder may develop in later years in one who has never been dependent on catheterisation. Similarly, obstructive uropathy at the bladder outlet may be identified at times only after the upper urinary tract has been seriously compromised. This, in the absence of bacteriuria. Persistent subclinical infection may enter by stealth and cause renal damage at any time.

The third class of complications may derive from the primary cord injury. Post-traumatic syringomyelia with consequent pain and increased disablement has been described by many authors. Sympathetic dysreflexia may intrude after years of freedom from this distressing syndrome. Pain of central origin though present at times from the onset may intrude after many years of exposure into the life of one who was initially pain-free, despite serious spinal cord damage. Sometimes 
this is concomitant with certain late complications, such as infection. Often it is unexplained. The fourth classification of late complications are of unclear aetiology and came to light from the mortality studies which have been conducted on the spinal cord injured population. Thus we observed increased incidence of death due to cancer, cardiovascular disease, respiratory disease and suicide, in comparison with the same causes of death in the general population. It is recognised that the above four common causes of death with the addition of accidents constitute the commonest causes of death in our society.

It appears that in the first wave of exaltation over the achievement of having reversed the mortality in spinal cord injured persons, we may have forgotten that these individuals remain seriously disabled and greatly at risk from many dire threats to their wellbeing. It thus behoves those of us who undertake the care of the spinal cord injured to ensure the continued provision of high quality monitoring and management and that it be provided in each community. To achieve this ideal we must also continue to educate family physicians, nurses and others who share responsibility for the care of the spinal cord injured patient who may be remote from an appropriate centre. Above all, we must educate the patient at every available opportunity for only thus will he be encouraged to maintain an intelligent self-interest in exercising caution essential for his wellbeing.

There is one final late complication which may be entitled 'Burn-out' which has become recognised amongst spinal cord injured persons as well as amongst members of the non-disabled population. It is manifest as a weariness and despondency which overwhelms the individual, frequently leading to retirement from the daily fray into a state of apathy and unconcern. Ongoing encouragement and support by a trusted medical attendant may postpone or prevent this exhaustion of the will and the spirit, which may be insidious and damaging. This could be the most important contribution of doctors treating spinal paralysis once the early phase of rehabilitation has been completed.

$$
\begin{aligned}
& \text { A. T. Jousse, M.D., } \\
& \text { Toronto, Canada. }
\end{aligned}
$$

